Trastornos depresivos en la infancia y adolescencia Depressive disorders in children and adolescents

\title{
J. Royo
}

\section{RESUMEN}

Los trastornos depresivos en niños y adolescentes presentan síntomas similares a los vistos en adultos, aunque la expresión de éstos puede variar significativamente en relación con el nivel de desarrollo evolutivo. Se trata de trastornos frecuentes, crónicos y recidivantes, en muchas ocasiones asociados a otros trastornos psiquiátricos comórbidos, y con un pobre pronóstico que puede mejorar con el diagnóstico precoz y el tratamiento. La valoración psiquiátrica puede ser difícil por lo que debe ser realizada por clínicos experimentados. Las investigaciones más recientes en relación con el tratamiento de estos trastornos se han centrado en la efectividad de la terapia cognitivo-conductual y de los inhibidores de la recaptación de serotonina.

Palabras clave. Depresión. Niños. Adolescentes. Diagnóstico. Tratamiento.

\begin{abstract}
Depressive disorders in children and adolescents are marked by core symptoms similar to those seen in adults, although symptom expression varies greatly with developmental stage. These disorders are common, chronic, and recurrent, and they are often associated with comorbid psychiatric conditions and poor prognosis that can be alleviated by early identification and treatment. The psychiatric assessment of depressed children and adolescents can be difficult and must be performed by an expert clinician. Recent research into the treatment of depression in children and adolescents has been focused on the effectiveness of cognitive-behavioural therapy and selective serotonin reuptake inhibitors.
\end{abstract}

Key words. Depression. Children. Adolescents. Diagnosis. Treatment.

ANALES Sis San Navarra 2002; 25 (Supl. 3): 97-103.

Psiquiatra. Hospital de Día Infanto-Juvenil de Salud Mental "Natividad Zubieta". Servicio Navarro de Salud. Pamplona.

\author{
Correspondencia: \\ J. Royo \\ Hospital de Día Infanto-Juvenil de Salud Mental \\ "Natividad Zubieta" \\ C/ San Cristóbal, $\mathbf{s} / \mathbf{n}$ \\ 31015 Pamplona \\ Tfno. 948136666 \\ E-mail: jroyom@intersep.org
}




\section{INTRODUCCIÓN}

Los trastornos afectivos (trastorno depresivo mayor, distimia y trastorno bipolar) que comienzan en los años de la infancia y la adolescencia se caracterizan por unas tasas familiares más altas de enfermedad afectiva que cuando los trastornos afectivos comienzan en edades más tardías, así como por unas tasas altas de enfermedades asociadas (en especial, trastornos de la conducta, ansiedad y déficit de atención), por un curso crónico recidivante de la enfermedad, con un deterioro a corto y largo plazo de la funcionalidad social e interpersonal, y por unas tasas aumentadas de abuso de sustancias y alto riesgo de suicidio consumado ${ }^{1}$. Por lo tanto, constituyen un importante capítulo dentro de la salud pública y es necesario su óptimo tratamiento tanto para la persona como para la familia y desde la perspectiva de salud de la población.

Actualmente, los trastornos depresivos, en los que vamos a centrar la exposición (trastorno depresivo mayor y trastorno distímico), se reconocen como uno de los principales trastornos psiquiátricos $\mathrm{y}$ un problema de salud mental en niños y adolescentes $^{2}$.

La práctica diagnóstica actual que utiliza los criterios del DSM-III-R (American Psychiatric Association, 1987) y DSM-IV (American Psychiatric Association, 1994), identifica unas tasas de prevalencia de los trastornos depresivos y bipolares que se pueden presentar con una relativa baja frecuencia en los años escolares, mostrando un ligero incremento de su prevalencia durante la adolescencia y alcanzando casi el nivel de las tasas de prevalencia en el adulto al final de la misma. Por ejemplo, las tasas de prevalencia de un trastorno depresivo mayor (DM) aumentan desde unas tasas en la infancia en torno al $1-2 \%$ hasta el 6-8\% al final de la adolescencia. Este incremento de la prevalencia a lo largo de la adolescencia probablemente está en relación con factores biológicos, ambientales y psicológicos. Las niñas parecen presentar más factores de riesgo para la depresión que los niños. Se cree que las tasas del trastorno distímico siguen un patrón similar, aunque los datos al respec- to no están tan bien demostrados ${ }^{3}$. En España se han estimado unas prevalencias de depresión mayor del $1,8 \%$, según criterios DSM-III en niños de 9 años, del 2,3\%, según criterios del DSM-III-R en adolescentes de 13 y 14 años $^{4}$ y del $3,4 \%$ en jóvenes de 18 años a partir de criterios CIE-10.

\section{PRESENTACIÓN CLÍNICA}

Cualquier niño puede sentirse triste en un momento determinado, pero para recibir el diagnóstico de episodio depresivo mayor debe cumplir los criterios diagnósticos de DSM-IV o CIE-10 (si utilizamos la clasificación internacional de enfermedades de la OMS). Aunque los criterios para diagnosticar un episodio depresivo mayor así como el trastorno distímico son los mismos en niños y adolescentes que en adultos, el cuadro clínico puede variar considerablemente según el nivel de desarrollo del niño. Por ejemplo, los niños generalmente presentan más síntomas de ansiedad, quejas somáticas, alucinaciones auditivas, rabietas y problemas de conducta. Conforme el nivel de desarrollo cognitivo va progresando, los niños de más edad pueden ser capaces de mostrar componentes cognitivos de su humor disfórico y baja autoestima, así como sentimientos de culpa y de infelicidad. Los adolescentes suelen manifestar más problemas de apetito y del sueño, delirios, ideación o intentos autolíticos, así como mayor repercusión funcional en su vida social y familiar que los niños. Además tienden a presentar menos síntomas neurovegetativos y quizás una mayor irritabilidad que los adultos con depresión mayor.

Muchos niños y adolescentes con depresión mayor presentan además otro trastorno psiquiátrico, siendo frecuente que se presenten dos o más trastornos comórbidos. Los diagnósticos comórbidos más frecuentes son el trastorno distímico (la llamada "depresión doble"), trastornos de ansiedad, trastornos de conducta y trastornos por uso de sustancias. El trastorno por ansiedad de separación es una comorbilidad habitual en niños pequeños. Los trastornos de personalidad, en especial el trastorno borderline de personalidad, son frecuentes, aunque algunos de los 
síntomas de estos trastornos pueden ser secundarios al trastorno depresivo.

Las relaciones familiares de los niños y adolescentes deprimidos suelen caracterizarse por la existencia de conflictos, maltrato, rechazo, y problemas de comunicación con pobre expresión de afecto positivo y apoyo. Los padres pueden estar también ellos mismos deprimidos o padecer otros trastornos psiquiátricos $\mathrm{u}$ otras enfermedades que condicionen una reducción de la efectividad de la educación parental. No obstante, estos problemas en el estilo educativo de los padres pueden ser secundarios a la interacción con un niño deprimido, irritable o desafiante.

Existe suficiente evidencia como para afirmar que experiencias adversas en la infancia (muerte o separación de los padres) incrementan el riesgo de depresión o ansiedad en la edad adulta.

\section{CURSO CLÍNICO}

La duración media de un episodio depresivo mayor es de 7 a 9 meses. Incluso con una buena respuesta al tratamiento, más de la mitad de los pacientes recaen. La recaída puede ser debida al curso natural de la enfermedad o bien debida al pobre cumplimiento o a la interrupción prematura del tratamiento. La mayoría de los episodios remiten entre 1 y 2 años después de su inicio, aunque un 6-10\% presenta un curso prolongado. La mitad de los pacientes presenta una recurrencia entre 1 y 2 años después del tratamiento, existiendo un $70 \%$ de recurrencia a los 5 años. La edad de comienzo, el número de episodios previos, la severidad del episodio actual, la presencia de comorbilidad psiquiátrica, el pobre cumplimiento terapéutico, la presencia de acontecimientos adversos (p. ej. conflicto familiar), la existencia de trastornos psiquiátricos en los padres y el deficiente funcionamiento social, son factores que pueden predecir un curso prolongado y la existencia de episodios recurrentes.

El trastorno bipolar se desarrolla en un 20-40\% de niños y adolescentes deprimidos con depresión mayor. Diferentes factores se asocian a una mayor vulnerabilidad al desarrollo de trastorno bipolar: comienzo precoz del episodio depresivo, enlentecimiento psi- comotor, síntomas psicóticos, historia familiar de trastorno bipolar o de depresión con síntomas psicóticos, y/o el desarrollo de hipomanía inducida farmacológicamente.

Los trastornos depresivos pueden afectar al desarrollo social, emocional y cognitivo, así como a la calidad del vínculo que se establece entre el niño y sus padres. Confieren, además, un mayor riesgo para las conductas suicidas y de abuso de sustancias, y repercuten en el funcionamiento sociofamiliar y académico del niño.

\section{DIAGNÓSTICO}

El diagnóstico se basa en una cuidadosa evaluación diagnóstica psiquiátrica, que debe incluir entrevistas con el niño, sus padres, y otros posibles informantes (profesores, pediatras, etc.). La valoración diagnóstica de niños y adolescentes deprimidos puede ser difícil. Debe ser llevada a cabo por clínicos experimentados ya que debe tenerse en cuenta el nivel de desarrollo evolutivo así como otros factores culturales (étnicos) que puedan influir en la presentación clínica. Los pacientes pueden tener dificultades para expresar sus sentimientos, o bien pueden mostrarse irritables y cooperar muy poco en la exploración.

Las entrevistas estandarizadas desarrolladas para fines de investigación ${ }^{5}$ son habitualmente demasiado extensas para poder ser utilizadas en la práctica clínica, requieren un alto nivel de adiestramiento y no son generalmente adecuadas para niños pequeños. Estas entrevistas estandarizadas aplicadas a niños de al menos 8 años de edad han obtenido una buena fiabilidad interentrevistador así como una buena fiabilidad test-retest, pero un bajo nivel de acuerdo entre la información de los padres y del niño. Este hecho no es sorprendente si tenemos en cuenta que los niños generalmente informan mejor sobre síntomas internalizantes (incluyendo ideación suicida), mientras que los padres son más conscientes de síntomas externalizantes como las dificultades de comportamiento $^{6}$. La información de los padres puede estar influida además por su propia psicopatología, por lo que es muy importante obtener información de otras fuentes (generalmente profesores). 
Los "checklists" o listados de síntomas derivados de las entrevistas estandarizadas y los listados de síntomas derivados del DSM-IV pueden ser herramientas más útiles para la práctica clínica, aunque no hay en la actualidad suficientes estudios que avalen su uso con fines diagnósticos.

Las escalas autoadministradas o administradas por el clínico, tales como el Beck Depression Inventory ${ }^{7}$, el Child Depression Inventory ${ }^{8}$ y el Center for Epidemiologic Studies Depression Scale ${ }^{9}$, entre otros, han sido diseñados para indagar acerca de síntomas depresivos. Sin embargo, debido a su baja especifidad, estas escalas no son útiles para diagnosticar una depresión clínica. Pueden ser, no obstante, usadas como screening de síntomas depresivos, para valorar la severidad del episodio depresivo o registrar la mejoría clínica.

En la década de los 70, los términos "depresión enmascarada y equivalente depresivo" conocieron su auge, que se mantuvo hasta bien entrada la década de los 90. El concepto de un trastorno psiquiátrico que enmascara u oculta otro trastorno psiquiátrico no es nuevo en psiquiatría infantil y toma su origen de los trabajos de Glaser $^{10}$, Cytryn y McKnew ${ }^{11}$ y Cytryn y col $^{12}$. La llamada depresión enmascarada hace referencia a una depresión que no muestra trastornos del ánimo, presentando otros síntomas como hiperactividad, conductas antisociales, conducta agresiva y trastornos del aprendizaje. El concepto de los equivalentes depresivos hace referencia a la presencia de quejas somáticas, fundamentalmente dolores (de barriga, de cabeza, etc.), como las que aparecen en la fobia escolar, en un niño que no muestra síntomas depresivos. Ahora es bien conocido que aquellos niños con depresión enmascarada y/o equivalentes depresivos realmente sí presentan síntomas depresivos y que la falta de detección de estos síntomas tiene que ver fundamentalmente con deficiencias en la exploración psicopatológica más que con la ausencia de síntomas depresivos.

\section{TRATAMIENTO}

El tratamiento de la depresión en niños $\mathrm{y}$ adolescentes comprende intervenciones psicoterapéuticas, sociofamiliares y farmacológicas. La planificación terapéutica se realizará sobre la base del diagnóstico, de la edad y de las características clínicas y sociofamiliares del paciente. La intervención incrementa su utilidad cuando se combinan diversos tipos de tratamiento. Una buena propuesta de modelo incluiría estrategias de intervención que incidan sobre las alteraciones afectivas, cognitivas, conductuales y biológicas, pero también en la familia y en la escuela. La hospitalización se recomienda cuando existe riesgo de suicidio o cuando existe una importante conflictividad familiar.

\section{Terapias cognitivo-conductuales}

Son probablemente el tipo de psicoterapia más usado. Reúnen un grupo de tratamientos, derivados en su mayoría del adulto, que incluyen terapia cognitiva, entrenamiento en habilidades sociales, entrenamiento en relajación, terapia racional emotiva, entrenamiento en resolución de problemas, intervenciones operantes, o intervenciones multi-componentes.

El tratamiento cognitivo-conductual se basa en la premisa de que los pacientes deprimidos tienen distorsiones cognitivas en el modo en que se ven ellos mismos, ven su entorno y el futuro, y en que estas distorsiones cognitivas contribuyen a su depresión.

El tratamiento cognitivo-conductual es eficaz tanto en poblaciones escolares con síntomas depresivos, como en adolescentes con depresión clínica y en la prevención de las recaídas ${ }^{13}$. La revisión literaria y el meta-análisis de los estudios localizados hechos por Reinecke y col ${ }^{14}$ apoyan la eficacia de estas intervenciones tanto a corto como a largo plazo. Según Jayson y col ${ }^{15}$ el éxito del tratamiento cognitivo-conductual está relacionado con la menor severidad del cuadro y con la menor edad de los adolescentes. Mayoritariamente los programas cognitivo-conductuales incluyen, por una parte el análisis cognitivo, con el reconocimiento de emociones, la relación de éstas con la conducta y la cognición y el cambio de atribuciones cognitivas negativas (aspectos de la terapia cognitiva de Beck). Por otra parte, incluyen la interven- 
ción más conductual, como el entrenamiento en habilidades sociales y la ayuda en la resolución de problemas.

La duración de los tratamientos cognitivo-conductuales no es larga (aproximadamente 12-20 sesiones).

La mayoría de estudios han encontrado una tasa alta de recaídas en el seguimiento cuando se aplica como única modalidad terapéutica.

\section{Terapia interpersonal}

Muy en relación con el modelo de intervención anterior, la terapia interpersonal es una psicoterapia breve que fue desarrollada para su uso con pacientes adultos depresivos. Está centrada en las relaciones sociales del individuo y el estado actual de las mismas. Los principales objetivos son disminuir la sintomatología depresiva y mejorar el funcionamiento interpersonal en el contexto de las interacciones.

El riesgo de recaídas parece ser bajo después de un tratamiento intensivo.

\section{Terapia psicodinámica}

La psicoterapia dinámica viene clínicamente explicitada, por algunos autores, según grupos de edad ${ }^{16}$.

Según estos autores durante la primera infancia la depresión implica a menudo disfunción familar y psicopatología paterna o materna, por lo que el terapeuta proporcionará a los padres apoyo, asesoramiento y educación en cuanto a la crianza del niño y un asesoramiento o psicoterapia para sus propios problemas.

En el niño preescolar, la terapia por el juego se considera la mejor intervención.

En el niño de edad escolar, principalmente en casos en que exista una resistencia y una falta de cooperación, la introducción de juegos estructurados y después menos estructurados ayudará en la terapia individual.

En la adolescencia la aparición del pensamiento abstracto y la defensa de intelectualización desplazan la transacción psicoterapéutica desde los juegos hasta intercambios verbales y la terapia hablada, siendo más parecido a la psicoterapia del adulto.

No existen publicaciones de estudios controlados, por lo que no se ha podido estimar con fiabilidad la frecuencia de recaídas.

\section{Tratamiento farmacológico}

La medicación antidepresiva en niños y adolescentes está indicada en casos de sintomatología depresiva severa que no ha respondido a intervenciones psicoterapéuticas. Está especialmente indicada si existe una notable repercusión sobre el funcionamiento escolar o académico. Asimismo es una clara indicación la existencia de riesgo autolítico ${ }^{17}$.

Para aquellos pacientes que requieren tratamiento farmacológico, los inhibidores selectivos de recaptación de la serotonina (ISRS) son el fármaco de elección, aunque la presencia de comorbilidad puede determinar la elección inicial de otros fármacos. Así, en un niño con depresión mayor y trastorno por déficit de atención con hiperactividad sería preferible iniciar tratamiento con un antidepresivo tricíclico, bupropion o venlafaxina antes que con un ISRS.

Dado la elevada tasa de recidivas, se recomienda la terapia de continuación para todos los pacientes durante al menos 6 meses. Los antidepresivos deben ser continuados a la misma dosis que se precisó para tratar la fase aguda del episodio depresivo. Al final de la fase de continuación, los pacientes que no requieran más tratamiento, deberán proceder a la retirada del fármaco progresivamente en el plazo de unas seis semanas. En los casos en los que el paciente deba seguir tomando tratamiento farmacológico o fase de mantenimiento (habitualmente en trastornos depresivos recurrentes), el clínico deberá considerar la duración (desde 1 año a indefinidamente).

No existe actualmente indicación para realizar pruebas de laboratorio antes o durante la administración de un ISRS. En el caso de utilizar antidepresivos tricíclicos, deberemos registrar periódicamente presión arterial, pulso periférico y peso. También es preciso en este caso la realización de un electrocardiograma previo al inicio 
de tratamiento y posteriores controles electrocardiográficos a lo largo de dicho tratamiento.

\section{Inhibidores selectivos de la recaptación de serotonina}

Estos fármacos han demostrado una buena efectividad en el tratamiento de adultos y jóvenes con depresión mayor, con un buen perfil de tolerabilidad (mínimos efectos secundarios), poca letalidad en sobredosis, y fácil administración (habitualmente una vez al día).

Dado que la mejoría con estos fármacos puede alcanzarse a las 4-6 semanas, los pacientes deben ser tratados con la dosis tolerable adecuada al menos durante este plazo de tiempo. Si no se consigue respuesta positiva, se procederá al incremento de la dosis o al cambio de medicación.

Los ISRS tienen una curva dosis-respuesta aplanada, es decir que la repuesta clínica máxima puede ser conseguida con dosis bajas del fármaco ${ }^{18}$.

En la tabla 1 se ofrece información con respecto a la dosis de ISRS utilizada en el tratamiento de un episodio depresivo mayor.

Los efectos secundarios de los ISRS son similares para todo el grupo, dosis-dependientes y suelen subsistir con el tiempo. Los ISRS pueden inducir "activación conductual" en la que los pacientes pueden mostrarse impulsivos, inquietos y/o agitados. Otros efectos secundarios incluyen síntomas gastrointestinales, inquietud, diaforesis, cefaleas, acatisia, y cambios en los patrones de sueño o apetito, así como dificultades en la función sexual.

La interrupción brusca de los ISRS con vida media corta, como paroxetina, puede inducir síntomas de abstinencia (síndrome de discontinuación) que emulan a los síntomas de una recaída. Estos síntomas pueden aparecer hasta 6-8 semanas después de la interrupción del fármaco.

\section{Antidepresivos tricíclicos}

No están recomendados actualmente como tratamiento de primera línea debido a su pobre eficacia y a la existencia de notables efectos secundarios.

Tabla 1. Dosis diarias totales sugeridas de ISRS en el tratamiento de niños y adolescentes.

\begin{tabular}{lccc}
\hline Medicación & Dosis inicial & Dosis diana inicial & Dosis máxima habitual \\
\hline Sertralina & $25 \mathrm{mg}$ & $50 \mathrm{mg}$ & $100-200 \mathrm{mg}$ \\
Fluoxetina & $5 \mathrm{mg}$ & $20 \mathrm{mg}$ & $20-60 \mathrm{mg}$ \\
Paroxetina & $10 \mathrm{mg}$ & $20 \mathrm{mg}$ & $20-40 \mathrm{mg}$ \\
Fluvoxamina & $50 \mathrm{mg}$ & $200 \mathrm{mg}$ & $200-300 \mathrm{mg}$
\end{tabular}

Nota: Estas dosis son sólo orientativas y proceden de la literatura disponible. Las dosis se ajustarán en cada caso, teniendo en cuenta los efectos secundarios y su eficacia.

\section{BIBLIOGRAFÍA}

1. HARRINGTON R. Annotation: the natural history and treatment of child and adolescent affective disorders. J Child Psychol Psychiatry 1992; 33: 1.287-1.302.

2. BiRMAHER B, RYAN N, WiLLIAMSON D. Depression in children and adolescents: clinical features and pathogenesis, in Mood Disorders Across the Life Span. En: Shulman K, Tohen M, Kutcher S. (eds.). Nueva York, Wiley, 1996; 51-83.

3. ANGOLD A. Childhood and adolescent depression, I: epidemiological and etiological aspects. Br J Psychiatry 1987; 152: 69-78.
4. Canals J, Martí-Heneberg C, Fernández J, DOMĖNECH E. A longitudinal study of depression in an urban Spanish pubertal population. Europ Child Adolesc Psychiatry 1995; 4: 102-111.

5. Costello A. Structured interviewing. En: Child and Adolescent Psychiatry: A Comprehensive Testbook, Lewis M, Ed. Baltimore: Williams \& Wilkins, 1995.

6. Cantwell DP, LeWinsohn PM, Rohde P, SEELEy JR. Correspondence between adolescent report and parent report of psychiatric diagnostic criteria. J Am Acad Child Adolesc Psychiatry 1997; 36: 610-619. 
7. Marton P, ChuRchard M, KUTCher S KoRENBLUM M. Diagnostic utility of the Beck Depression Inventory with adolescents psychiatric outpatients and inpatients. Can J Psychiatry 1991; 36: 428-431.

8. Kovacs M. Children's Depression Inventory (CDI) Manual. North Tonawanda, NY: MultiHealth Systems, 1992.

9. Roberts RE, LEWINSOHN PM, SEELEY JR. Screening for adolescent depression: a comparison of depression scales. J Am Acad Child Adolesc Psychiatry 1991; 30: 58-66.

10. GLASER K. Masked depression in children and adolescents. Annual Progress Child Psychiatry Child Dev 1968; 1: 345-355.

11. Cytryn L, McKnew DH. Proposed classification of chilhood depression. Amer J Psychiatry 1972; 129, 149-55.

12. CytRYn L, McKnew DH, BunNey WE. Diagnosis of depression in children: a reassessment. American J Psychiatry 1980; 137, 22-5.

13. Birmaher B, RyAn ND, Williamson DE, BRENT DA, Kaufman $J$ et al. Childhood and adolescent depression: A review of the past
10 years. Part II. J Am Acad Child Adolesc Psychiatry 1996; 35: 1575-1583.

14. ReineCKe MA, RYAn NE, Dubois DL. Cognitivebehavioral therapy of depression and depressive symptoms during adolescence: A review and meta-analysis. J Am Acad Child Adolesc Psychiatry 1998; 37: 26-34.

15. Jayson D, WoOd A, Kroll L, Fraser J, HARRINGTON R. Wich depressed patient respond to cognitive-behavioral treatment? J Am Acad Child Adolesc Psychiatry 1998; 37: $35-40$.

16. ShafII M, LEe ShafII S. Psicoterapia dinámica de la depresión. En: M Shafii, editor. Barcelona. Martínez Roca, 1994; 142-156.

17. Ambrosini P, Bianchi M, Rabinovich H, Eha J. Antidepressant treatments in children and adolescents. I. Affective Disorders. J Am Acad Child Adolesc Psychiatry 1993; 32: 1-6.

18. Practice parameters for the assesment and treatment of children and adolescents with depressive disorders. J Am Acad Child Adolesc Psychiatry 1998; 37 (10 Suppl.): 63S83S. 
